\title{
Semaphorin 4D promotes bone invasion in head and neck squamous cell carcinoma
}

\author{
HIROYUKI TAKADA $^{1}$, SOICHIRO IBARAGI ${ }^{1}$, TAKANORI EGUCHI ${ }^{2,3}$, TATSUO OKUI ${ }^{1}$, \\ KYOICHI OBATA ${ }^{1}$, MASANORI MASUI ${ }^{1}$, AYAKA MORISAWA ${ }^{1}$, KIYOFUMI TAKABATAKE ${ }^{4}$, \\ HOTAKA KAWAI $^{4}$, NORIE YOSHIOKA ${ }^{1}$, NUR MOHAMMAD MONSUR HASSAN ${ }^{5}$, \\ TSUYOSHI SHIMO ${ }^{1,3}$, GUO-FU HU ${ }^{6}$, HITOSHI NAGATSUKA ${ }^{4}$ and AKIRA SASAKI ${ }^{1}$
}

\begin{abstract}
Departments of ${ }^{1}$ Oral and Maxillofacial Surgery, and ${ }^{2}$ Dental Pharmacology, Okayama University Graduate School of Medicine, Dentistry, and Pharmaceutical Sciences, Kita-ku; ${ }^{3}$ Advanced Research Center for Oral and Craniofacial Sciences, Okayama University Dental School; ${ }^{4}$ Departments of Oral Pathology and Medicine, Okayama University Graduate School of Medicine, Dentistry, and Pharmaceutical Sciences, Kita-ku, Okayama, Japan; ${ }^{5}$ School of Dentistry and Health Sciences, Charles Sturt University, Orange NSW, Australia; ${ }^{6}$ Molecular Oncology Research Institute, Tufts Medical Center, Boston, MA, USA
\end{abstract}

Received April 2, 2017; Accepted June 15, 2017

DOI: $10.3892 /$ ijo.2017.4050

\begin{abstract}
Head and neck squamous cell carcinomas (HNSCCs) frequently invade the bones of the facial skeleton. Semaphorin 4D (Sema4D) is an axon guidance molecule produced by oligodendrocytes. Sema4D was also identified in the bone microenvironment and many cancer tissues including HNSCC. To date, however, the role of Sema4D in cancer-associated bone disease is still unknown. This is the first study to demonstrate the role of Sema4D in bone invasion of cancer. In the clinical tissue samples of bone lesion of HNSCC, Sema4D was detected at high levels, and its expression was correlated with insulin-like growth factor-I (IGF-I) expression. In vitro experiments showed that IGF-I regulates Sema4D expression and Sema4D increased proliferation, migration and invasion in HNSCC cells. Sema4D also regulated the expression of receptor activator of nuclear factor $\kappa \beta$ ligand (RANKL) in osteoblasts, and this stimulated osteoclastgenesis. Furthermore, knockdown of Sema4D in HNSCC cells inhibited tumor growth and decreased the number of osteoclasts in a mouse xenograft model. Taken together, IGF-I-driven production of Sema4D in HNSCCs promotes osteoclastogenesis and bone invasion.
\end{abstract}

Correspondence to: Dr Soichiro Ibaragi, Department of Oral and Maxillofacial Surgery, Okayama University Graduate School of Medicine, Dentistry, and Pharmaceutical Sciences, 2-5-1 Shikata-cho, Kita-ku, Okayama 700-8525, Japan

E-mail: sibaragi@md.okayama-u.ac.jp

Key words: semaphorin 4D, head and neck squamous cell carcinoma, bone invasion, osteoclastogenesis, Plexin-B1, insulin-like growth factor-I

\section{Introduction}

Head and neck squamous cell carcinomas (HNSCCs) arise from epithelia of the oral cavity, pharynx, larynx, nasal cavity or paranasal sinuses, and they frequently invade the surrounding bones that comprise the facial skeleton because of the anatomical proximity (1). The clinical importance of bone invasion of HNSCC includes a high morbidity rate and a worse prognosis. It is thus essential to control bone invasion in the treatment of HNSCC (1).

The progression of HNSCC cells from epithelia to bone induces the production of cancer-associated factors synthesized by either HNSCC cells or stromal cells activated by HNSCC cells (2). These factors induce the expression of receptor activator of nuclear factor $\kappa \beta$ ligand (RANKL) in the osteoblasts, which results in osteoclast activation and bone destruction. Bone destruction leads to the release of growth factors preserved in bone, including insulin-like growth factor-I (IGF-I) and transforming growth factor- $\beta$ (TGF- $\beta$ ) $(3,4)$. These growth factors promote HNSCC bone invasion and further increase the production of cancer-associated factors, which triggers a vicious circle (2).

Semaphorins are a large family of molecules containing a cysteine-rich sema domain. They were originally shown to regulate axonal growth cone guidance in the developing central nervous system (5-7). To date, $>30$ semaphorins have been identified and classified into eight subgroups based on their species of origin and sequence similarity. Semaphorin receptors, i.e., the plexins, also contain a sema domain. Nine known plexins, i.e., Plexin-A1 to -A4, -B1 to -B3, -C1, and -D1 are grouped according to their structure similarity. Semaphorin-plexin interactions were initially thought to be limited to the central nervous system, but these interactions are now implicated widely in the non-nervous systems, i.e., the cardiovascular (8), endocrine (9), gastrointestinal (10), immune (11) and respiratory (12) systems. 
Semaphorin 4D (Sema4D) is an axon guidance molecule produced by oligodendrocytes (13). Sema4D is also expressed by T-lymphocytes and eosinophils and functions in the immune system (13). Sema4D was identified in the bone microenvironment, and shown to be expressed by osteoclasts that act through Plexin-B1 on osteoblasts to inhibit their differentiation through inhibiting IGF-I signaling (14). Sema4D is also highly expressed in many cancer tissues including cancer of the prostate, colon, breast, lung and HNSCC (15). Moreover, Sema4D was recently reported to promote osteoclastogenesis and bone metastasis in breast cancer (7).

These findings prompted us to explore whether HNSCC cells could use Sema4D to invade bone. The results of our present study demonstrate that 1) a high amount of Sema4D was present in the tissue samples of bone lesions of HNSCC, and 2) the expression of Sema4D was correlated with IGF-I expression. We also observed that 3) HNSCC cell lines express high levels of Sema4D driven by IGF-I, and 4) Sema4D increases the proliferation, migration and invasion of HNSCC cells. Our results demonstrate that 5) osteoblasts produce RANKL in response to Sema4D, which activates osteoclasts, further stimulating bone resorption. Finally, we observed 6) the inhibition of tumor growth and decreased numbers of osteoclasts in a mouse model of HSC-2 cells with knocked down Sema4D compared to controls.

\section{Materials and methods}

Clinical samples. All of the HNSCC patients whose cases were retrospectively examined herein were treated at the Department of Oral and Maxillofacial Surgery, Okayama University Hospital (Okayama, Japan) between 2000 and 2010, and the diagnosis was pathologically confirmed HNSCC (six cases of soft tissue HNSCC, include three cases of T4NOM0, two cases of T4N1M0 and a case of T4N2M0. Nine cases of HNSCC with jaw bone invasion include four cases of T4N0M0, two cases of T4N1M0 and three cases of T4N2M0). No patient had received chemotherapy or radiation therapy before surgery. The study was approved by the ethics committee of the Okayama University Graduate School of Medicine, Dentistry, and Pharmaceutical Sciences (protocol no. 1949), including the use of an advertisement poster in place of patient consent. Written consent was not acquired for this retrospective study.

We had access to the patient records prior to the data anonymization. The surgically resected tissues were collected as part of the patients' routine care by the authors. All patient information was anonymized and de-identified prior to the analysis. The sections were deparaffinized and then autoclaved in $0.2 \%$ citrate buffer for $15 \mathrm{~min}$ for antigen retrieval. Sections were incubated with $3 \%$ hydrogen peroxide for $30 \mathrm{~min}$ to block endogenous peroxidase activity.

Primary rabbit anti-human Sema4D polyclonal IgG, rabbit anti-human Plexin-B1 polyclonal IgG, rabbit anti-human IGF-I polyclonal IgG and rabbit anti-human IGFI-R polyclonal IgG antibodies (Abcam, Cambridge, MA, USA) were used for immunohistochemisty analyses. The specimens were incubated with a 1:100 dilution of the antibody overnight at $4^{\circ} \mathrm{C}$, followed by three washes with Tris-buffered saline (TBS). The slides were then treated with a streptoavidin-biotin complex (Envision System Labeled Polymer, horseradish peroxidase
(HRP); Dako, Carpinteria, CA, USA) for $60 \mathrm{~min}$ at a dilution of 1:100. The immunoreaction was visualized using a 3,3'-diaminobenzidine (DAB) substrate-chromogen solution (Dako Cytomation Liquid DAB Substrate Chromogen System, Dako). Finally, the sections were immersed in an ethanol and xylene bath and mounted for examination. Sections were photographed and the DAB density was detected using ImageJ (NIH, Bethesda, MD, USA). Thereafter, the threshold of density was set automatically and the $\alpha$ value $(0-255)$ was calculated also by ImageJ software.

Cell culture and reagents. Human HNSCC cell lines HSC-2 and SAS and bone marrow-derived stroma cell line ST-2 cells were obtained from the Cell Engineering Division of RIKEN BioResource Center (Tsukuba, Ibaraki, Japan). Both cancer cell lines were cultured in Dulbecco's modified Eagle's medium/Ham's F-12 nutrient mixture (DMEM/F-12) supplemented with $10 \%$ fetal bovine serum (FBS). The bone marrow cells were isolated from 5-week-old male C57BL/6 mice obtained from Charles River (Yokoyama, Japan).

Bone marrow cells was cultured in the $\alpha$-modification of minimum essential medium ( $\alpha$ MEM; Sigma, St. Louis, MO, USA) supplemented with $10 \%$ fetal calf serum (FCS) (Hyclone Laboratories, Logan, UT, USA) and penicillin/streptomycin solution in an atmosphere of $5 \% \mathrm{CO}_{2} /$ air at $37^{\circ} \mathrm{C}$. Linsitinib (an IGF-I receptor inhibitor), SCH772984 (an ERK inhibitor) and MK2206 (an AKT inhibitor) were purchased from MedChem Express (Princeton, NJ, USA). Primary mouse osteoblasts (i.e., osteoblasts from a cranial bone culture kit F-2) were purchased from Cosmo Bio (Tokyo, Japan) and cultured in $\alpha \mathrm{MEM}$ supplemented with $10 \%$ FCS.

Immunoblot analysis. Cell monolayer cultures were rinsed with ice-cold phosphate-buffered saline (PBS) and lysed in an ice-cold lysis buffer $(50 \mathrm{mM}$ Tris-HCl, $\mathrm{pH} 7.4$, containing $150 \mathrm{mM} \mathrm{NaCl}, 1 \%$ Triton X-100, $1 \%$ NP-40, $10 \mathrm{mM} \mathrm{NaF}$, $100 \mathrm{mM}$ leupeptin, $2 \mathrm{mg} / \mathrm{ml}$ aprotinin and $1 \mathrm{mM}$ phenylmethyl sulfonyl fluoride). Protein quantification was performed by Protein Assay System (Thermo Fisher Scientific, Waltham, MA, USA). Cell lysates containing $10 \mu \mathrm{g}$ of total protein in the lysis buffer were electrophoresed in $12 \%$ sodium dodecyl sulfate-polyacrylamide gel electrophoresis (SDS-PAGE) gels, and the proteins were then transferred to nylon membranes (Immobilon-P; Millipore, Bedford,MA,USA). The membranes were blocked with $2 \%$ non-fat dry milk in TBS overnight at $4^{\circ} \mathrm{C}$ and then incubated with rabbit anti-human Sema4D polyclonal IgG, rabbit anti-human Plexin-B1 polyclonal IgG (Abcam) or rabbit anti-mouse RANKL polyclonal IgG (Santa Cruz Biotechnology, Santa Cruz, CA, USA) antibodies used at a 1:200 dilution. HRP-conjugate of goat anti-rabbit polyclonal $\mathrm{IgG}$ was used as the secondary antibody at a 1:1000 dilution (Amersham Biosciences, Buckinghamshire, UK). Bands were visualized by the ECL chemiluminescence detection method (RPN2109; Amersham Biosciences).

Cell proliferation. Cells were seeded at a density of $3 \times 10^{3}$ cells per well in a 96-well plate and cultured for $24 \mathrm{~h}$. They were then cultured in the presence of recombinant human Sema4D (R\&D Systems, Minneapolis, MN, USA). An MTS assay was performed to obtain a relative cell number after $48 \mathrm{~h}$ of 
incubation under the experimental procedure specified by the manufacturer (Cell Titer 96 AQueous One Solution Cell Proliferation Assay; Promega, Madison, WI, USA).

Invasion and migration assay. We studied the invasion and migration of cells by using Boyden chambers with and without Matrigel $^{\circledR}$ (BD Biosciences, Franklin Lakes, NJ, USA). Cells in the logarithmic growth phase were detached by trypsinEDTA, and $3 \times 10^{4}$ cells in medium without FBS were added to polycarbonate membranes (pore size $8.0 \mu \mathrm{m}$ ). Sema4D was added to the lower chamber, and the system was incubated at $37^{\circ} \mathrm{C}$ for $24 \mathrm{~h}$ in $5 \% \mathrm{CO}_{2}$. After incubation and fixation, the cells on the upper side of the membrane were removed with a cotton swab and the remaining cells were stained with $2 \%$ Crystal Violet (Sigma). The number of stained cells on the lower side of the membrane in four microscopic fields was counted, and the average of cells in three wells was determined.

TRAP staining and osteoclast activity assay. Bone marrow macrophages (BMMs) were prepared as previously described (16). Bone marrow cells were isolated from 5-week-old male C57BL/6 mice obtained from Charles River and cultured with $10 \mathrm{ng} / \mathrm{ml}$ of macrophage colonystimulating factor (M-CSF; R\&D Systems) for $24 \mathrm{~h}$, floating cells were collected and cultured with $30 \mathrm{ng} / \mathrm{ml}$ M-CSF for 3 days. Thereafter, cells that were attached to the plates were collected as BMMs.

BMMs $\left(4 \times 10^{4}\right)$ were seeded on 48 -well plates and cultured with $30 \mathrm{ng} / \mathrm{ml} \mathrm{M-CSF}$ in the presence or absence of Sema4D (R\&D Systems) and/or $50 \mathrm{ng} / \mathrm{ml}$ RANKL (Peprotech, London, UK) for 6 days. The medium was completely changed every 2 days. The cells were then fixed and stained for TRAP (Sigma), and the number of TRAP-positive multinucleated cells (nuclear number $>3$ ) in each well was counted.

For the osteoclast activity assay, BMMs $\left(1 \times 10^{5}\right)$ were seeded on hydroxyapatite-coated plates (Osteo Assay Surface 24-well plate, Corning, NY, USA) and cultured as above. The area of pits on the plates was quantified by ImageJ. For RANKL inhibitory assay, BMMs $\left(1 \times 10^{5}\right)$ were seeded on 24-well plates and cultured without RANKL with $30 \mathrm{ng} / \mathrm{ml} \mathrm{M}-\mathrm{CSF}$ in the presence or absence of Sema4D and osteoprotegerin (OPG; Peprotech) for 6 days. The number of TRAP-positive multinucleated cells (nuclear number $>3$ ) in each well was counted.

Knockdown of Sema4D in HNSCC cells. We purchased Sema4D short hairpin RNAs (shRNAs) cloned into a pLKO.1-puro plasmid under the control of human phosphoglycerate kinase eukaryotic promoter for stable expression (Mission $^{\circledR}$ shRNA Plasmid DNA, SHCLND-NM_006378, Sigma) and scrambled sequence shRNA constructs (Mission Non-Target shRNA Control Vector, SHC002, Sigma). HNSCC cells are transfected with shRNA-pLKO.1-puro using Lipofectamine $3000{ }^{\circledR}$ (Thermo Fisher Scientific) according to the manufacturer's instructions.

After 2 weeks in medium containing the selection antibiotic puromycin, cells stably expressing the shRNA were selected from transfected cultures. Sema4D knockdown was confirmed by performing an immunoblot analysis and evaluated by using ImageJ. The five types of shRNA used were: shSema4D\#1, CCGGGCCTGTGACTTGGTTTCTCTTCT
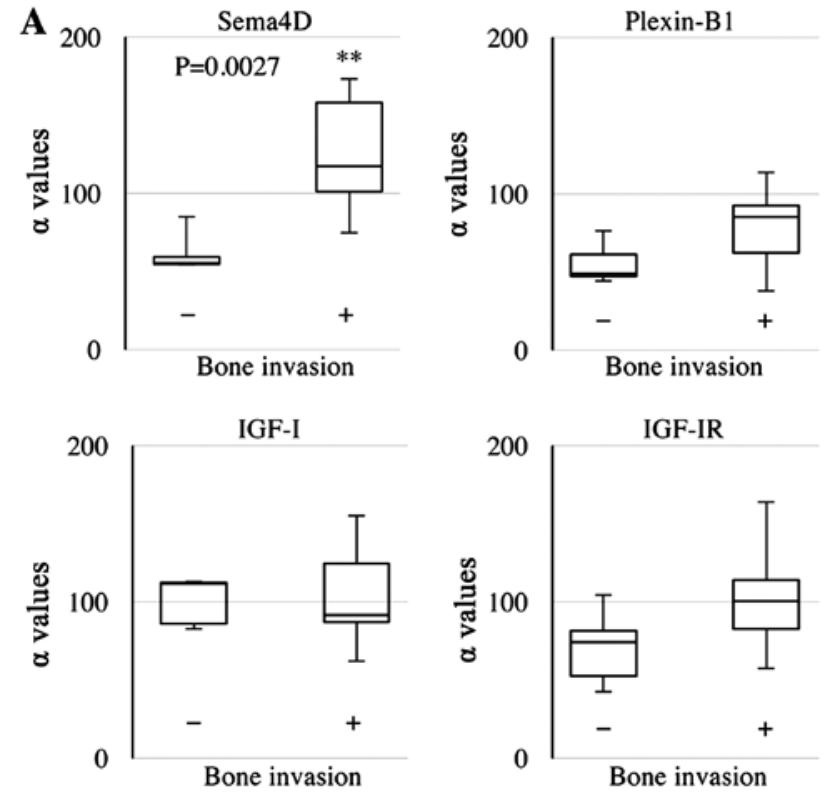

B

\begin{tabular}{|l|r|r|r|r|}
\hline & Sema4D & Plexin-B1 & \multicolumn{1}{c|}{ IGF-I } & IGF-IR \\
\hline Sema4D & 1 & 0.16 & 0.52 & 0.04 \\
\hline Plexin-B1 & & 1 & 0.27 & -0.08 \\
\hline IGF-I & & & 1 & -0.07 \\
\hline IGF-IR & & & & 1 \\
\hline
\end{tabular}

Figure 1. Expression levels of Sema4D, Plexin-B1, IGF-I and IGF-IR in clinical HNSCC samples. (A) $\alpha$ values (0-255) calculated from DAB density in tumor samples (six cases of soft tissue HNSCC and nine cases of HNSCC with jaw bone invasion). ${ }^{* *} \mathrm{p}<0.01$. (B) Correlation coefficients showing the relationship between pairs of groups of Sema4D, Plexin-B1, IGF-I and IGF-IR.

CGAGA AGAGA A ACCA AGTCACAGGCT T T T TG; shSema4D\#2, CCGGCGAACCAAAGATCGTCATCAAC TCGAGTTGATGACGATCTTTGGTTCGTTTTTG; shSema4D\#3, CCGGGCCTGTGTTCTATGCACTCTTCT CGAGAAGAGTGCATAGA ACACAGGCT T T T TG; shSema4D\#4, CCGGCCTGAACTTAACATCCTTTAA CTCGAGTTAAAGGATGTTAAGTTCAGGTTTTTG; and shSema4D\#5, CCGGGTACGGTCTTATGGGCAGAAA CTCGAGTTTCTGCCCATAAGACCGTACTTTTTG.

Bone xenograft model. All animal experiments were approved by the Institutional Animal Care and Use Committee of Okayama University. The bone xenograft model was prepared as previously described (17). Five-week-old male athymic mice (nu/nu) were obtained from CLEA Japan (Tokyo). HSC-2 cells, $5 \times 10^{6}$ per mouse, were inoculated into the paraperiosteal tissue of the tibial metaphysis (17). Six mice per group were used.

Tumor sizes and body weights were measured weekly, and the former were recorded in cubic millimeters (length $\mathrm{x}$ width ${ }^{2} / 2$ ). The animals were sacrificed at day 28 , and the tumor tissues were removed and weighed.

Immunohistochemistry for xenograft tumor specimens. Paraffin blocks of specimens were cut at $4 \mu \mathrm{m}$ thickness. Immunohistochemistry (IHC) was performed with rabbit 
A
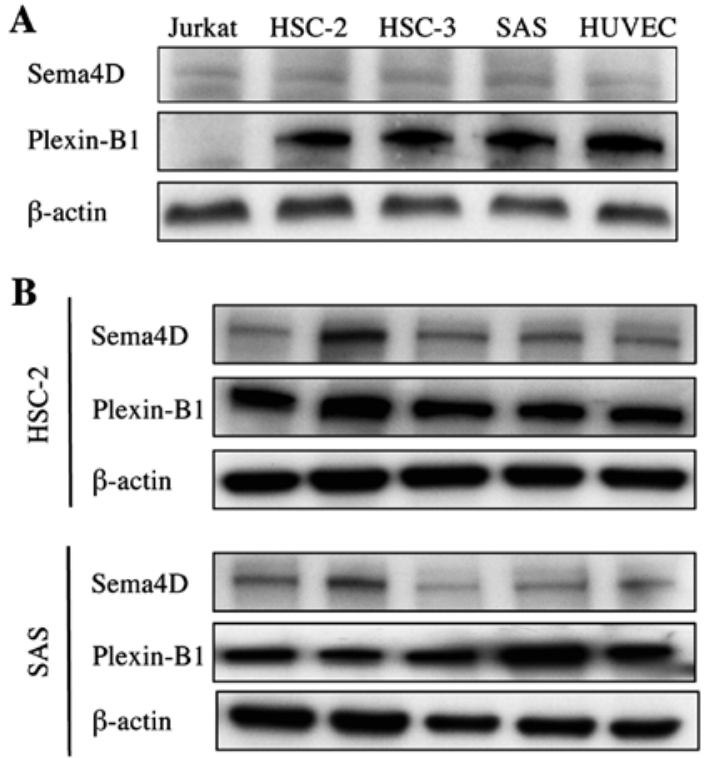

$\begin{array}{lccccc}\text { IGF-I } & - & + & + & + & + \\ \text { Linsitinib } & - & - & + & - & - \\ \text { SCH772984 } & - & - & - & + & - \\ \text { MK2206 } & - & - & - & - & +\end{array}$

Figure 2. Expression of Sema4D and Plexin-B1 in HNSCC cell lines. (A) Expression of Sema4D and Plexin-B1 in HSC-2, HSC-3 and SAS cells. Jurkat and HUVEC were the positive controls of Sema4D and Plexin-B1, respectively. (B) Expression of Sema4D and Plexin-B1 induced by IGF-I in HSC-2 and SAS cells. Cells were cultured in the presence or absence of IGF-I treated with Linsitinib, SCH772984 or MK2206 at the concentration of $1 \mu \mathrm{M}$. Similar results were obtained in at least 3 independent experiments.

anti-human Sema4D polyclonal IgG, rabbit anti-human Plexin-B1 polyclonal IgG, rabbit anti-human IGF-I polyclonal $\mathrm{IgG}$, rabbit anti-human IGFI-R polyclonal IgG, rabbit antihuman Ki67 polyclonal IgG and rabbit anti-mouse CD31 polyclonal IgG antibodies used at a 1:100 dilution (Abcam). Sections were incubated with the primary antibodies at $4^{\circ} \mathrm{C}$ for $16 \mathrm{~h}$, and visualized with the Envision system (Dako). The number of TRAP-positive multinucleated osteoclasts in three visual fields under a microscope (x200) were counted, and the osteoclast number per bone perimeter (per $100 \mathrm{~mm}$ ) was calculated as previously described (18).

Statistical analysis. We analyzed the data (except those from the clinical samples) by using the unpaired Student's t-test for comparisons of two groups, and Fisher's protected least significant difference (Fisher's PLSD) for multiple group comparisons. Results are expressed as the mean $\pm \mathrm{SD}$. Values of $p<0.05$ were considered significant. The data from clinical samples were analyzed using the Mann-Whitney U test because of the non-parametric distribution of data. The relationships between pairs of groups were analyzed by using the calculated correlation coefficient.

\section{Results}

Sema4D is highly expressed in the HNSCC tumors with bone invasion and correlates with IGF-I expression. We examined the expression of Sema4D in different HNSCC tumors, and
A

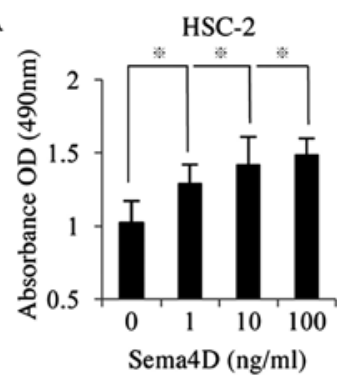

C

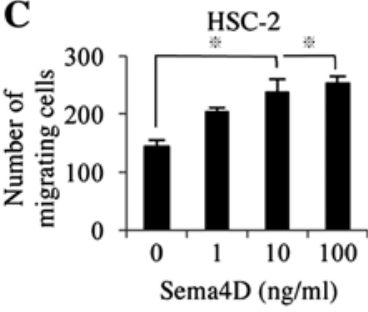

$\mathbf{E}$

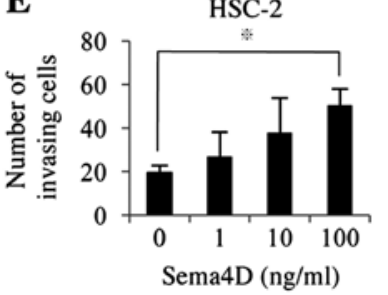

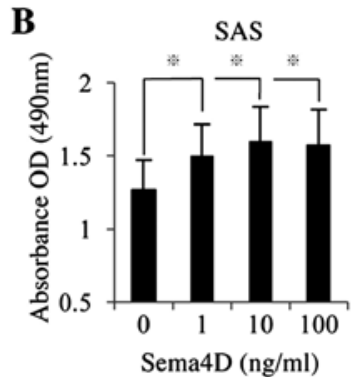

D

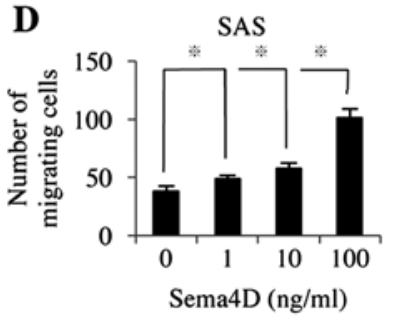

$\mathbf{F}$

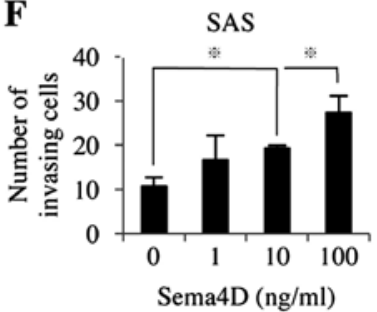

Figure 3. Effects of Sema4D on cellular activity of HSC-2 and SAS cells. (A and B) The MTS assay was performed to obtain the relative cell number. (C and D) Migration was studied by using Boyden chambers. (E and F) Invasion was studied using Boyden chambers with Matrigel ${ }^{\circledR}$. All experiments were repeated three times. Data are the mean \pm SD of triplicates from a typical experiment. " $\mathrm{p}<0.05$.

found that its level was 2.1-fold higher in HNSCCs with bone invasion compared to HNSCCs without bone invasion ( $p=0.0027$ ) (Fig. 1A). Of note, Sema4D expression was significantly correlated with IGF-I expression (correlation coefficient: 0.52) (Fig. 1B).

Sema4D and Plexin-Bl are expressed in HNSCC cells in vitro. Since Sema4D was highly expressed in HNSCC tumors with bone invasion, we examined whether Sema4D was expressed in HNSCC cell lines HSC-2, HSC-3 and SAS in vitro. All three HNSCC cell lines exhibited weak Sema4D expression, but this expression was comparable to that of the Jurkat $\mathrm{T}$ cells used as the positive control for Sema4D (Fig. 2A).

Plexin-B1 was strongly expressed in all three HNSCC cell lines similarly to HUVEC cells, which were the positive control for Plexin-B1 (Fig. 2A). Noteworthy, IGF-I increased the expression of Sema4D by 2.1- and 1.5-fold in HSC-2 and SAS cells, respectively (Fig. 2B). Moreover, Linsitinib (an IGF-IR inhibitor), SCH772984 (an ERK inhibitor) and MK2206 (an AKT inhibitor) suppressed IGF-I-induced Sema4D expression. Taken together, our findings demonstrate that Sema4D and Plexin-B1 were expressed in HNSCC cells and that Sema4D expression was regulated by IGF-IR downstream signaling including ERK and AKT. TGF- $\beta$ did not increase Sema4D expression in HSC-2 or SAS cells (data not shown). 
A

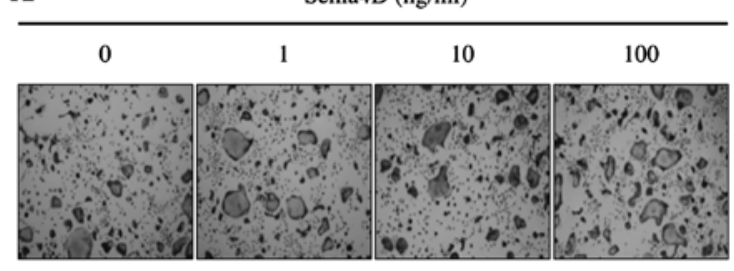

B

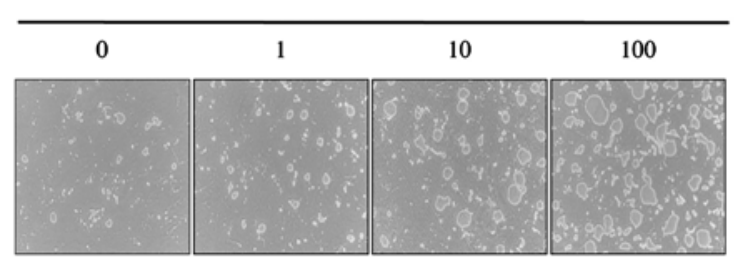

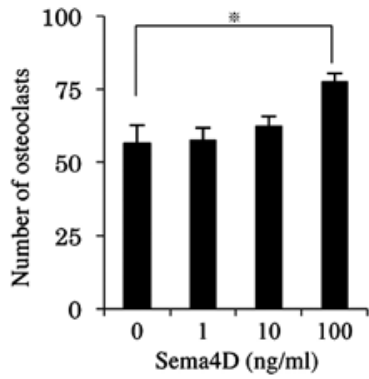

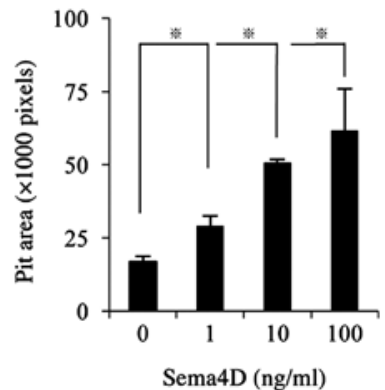

C
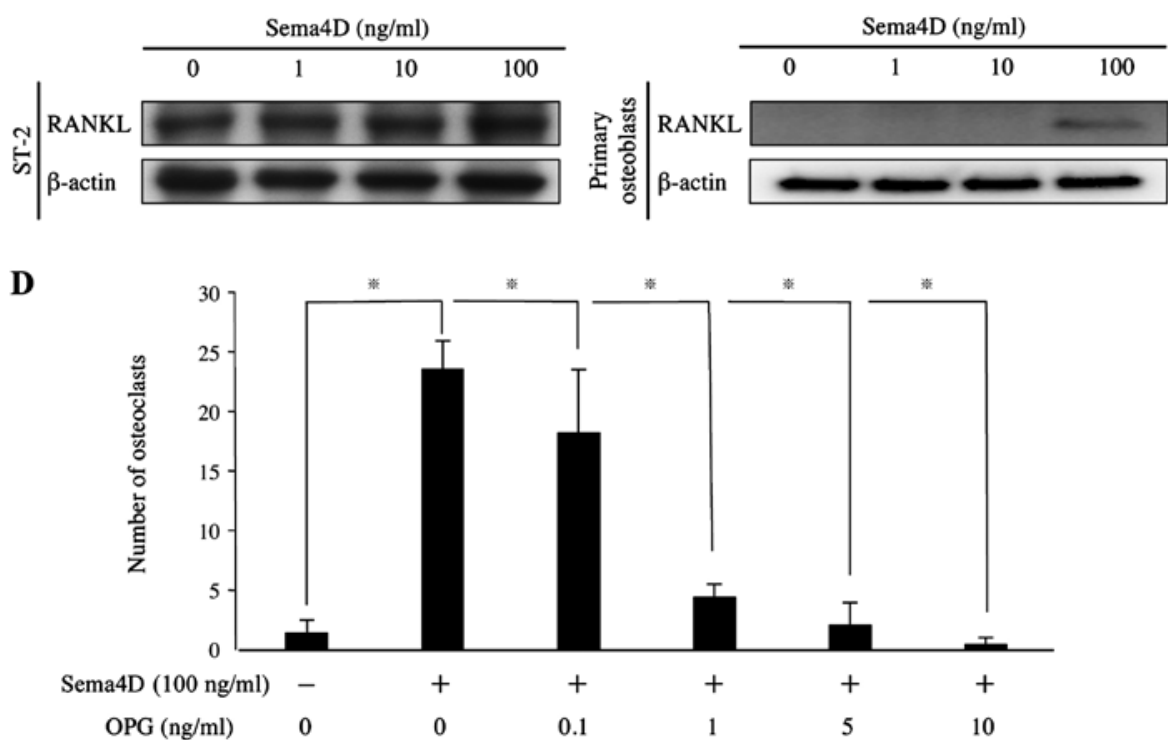

Figure 4. Effects of Sema4D on the formation and activity of osteoclasts in vitro. (A) BMMs were cultured with RANKL and M-CSF in the presence or absence of Sema4D. The number of TRAP-positive multinucleated cells in each well was counted. (B) BMMs were cultured on hydroxyapatite-coated plates. The area of pits on the plates was quantified by ImageJ. (C) ST-2 cells and primary mouse osteoblasts were cultured with Sema4D. RANKL expression was examined by immunoblot analysis. (D) BMMs were cultured without RANKL with M-CSF in the presence or absence of Sema4D (100 ng/ml) and OPG. The number of TRAP-positive multinucleated cells in each well was counted. All experiments were repeated three times. Data are the mean \pm SD of triplicates from a typical experiment. ${ }^{*} \mathrm{p}<0.05$.

Sema4D upregulates HNSCC cellular activity. To examine the effect of Sema4D in vitro, we treated HNSCC cells with different concentrations of Sema4D and measured cell proliferation by MTS assay. With $100 \mathrm{ng} / \mathrm{ml} \mathrm{Sema4D,} \mathrm{the}$ proliferation was stimulated 1.5 -fold and 1.3-fold (Fig. 3A and $\mathrm{B}$ ) and the cell migration was enhanced 1.8 -fold and 2.7-fold in HSC-2 and SAS cells, respectively (Fig. 3C and D). With $100 \mathrm{ng} / \mathrm{ml}$ Sema4D, invasion was enhanced 2.6-fold and 2.4-fold in HSC-2 and SAS cells, respectively (Fig. 3E and F).

Sema $4 D$ enhances osteoclastogenesis and bone resorptive activity. Since Sema4D increased proliferation of HNSCC cell lines, we next studied the effect of Sema4D on osteoclasts. BMMs were treated with Sema4D in the presence of M-CSF and RANKL for 6 days. At the concentration of $100 \mathrm{ng} / \mathrm{ml}$, Sema4D increased the osteoclastogenesis by 1.4 -fold and the osteolytic activity by 3.8 -fold (Fig. 4A and B). Since Plexin-B1 is known to be expressed in osteoblasts (14), we postulated that Sema4D enhances osteoclastogenesis through Plexin-B1 on osteoblasts. To test this hypothesis, we treated ST-2 cells and primary mouse osteoblasts with Sema4D and then evaluated the expression of RANKL by immunoblotting. As shown in Fig. 4C, RANKL expression was upregulated by $100 \mathrm{ng} / \mathrm{ml}$ Sema4D 1.5-fold in ST-2 cells, and was induced in primary mouse osteoblasts. To elucidate the role of RANKL in Sema4D-stimulated osteoclastogenesis, we treated BMMs with Sema4D without exogenous soluble RANKL with different concentrations of OPG. Fig. 4D shows that Sema4D promoted 

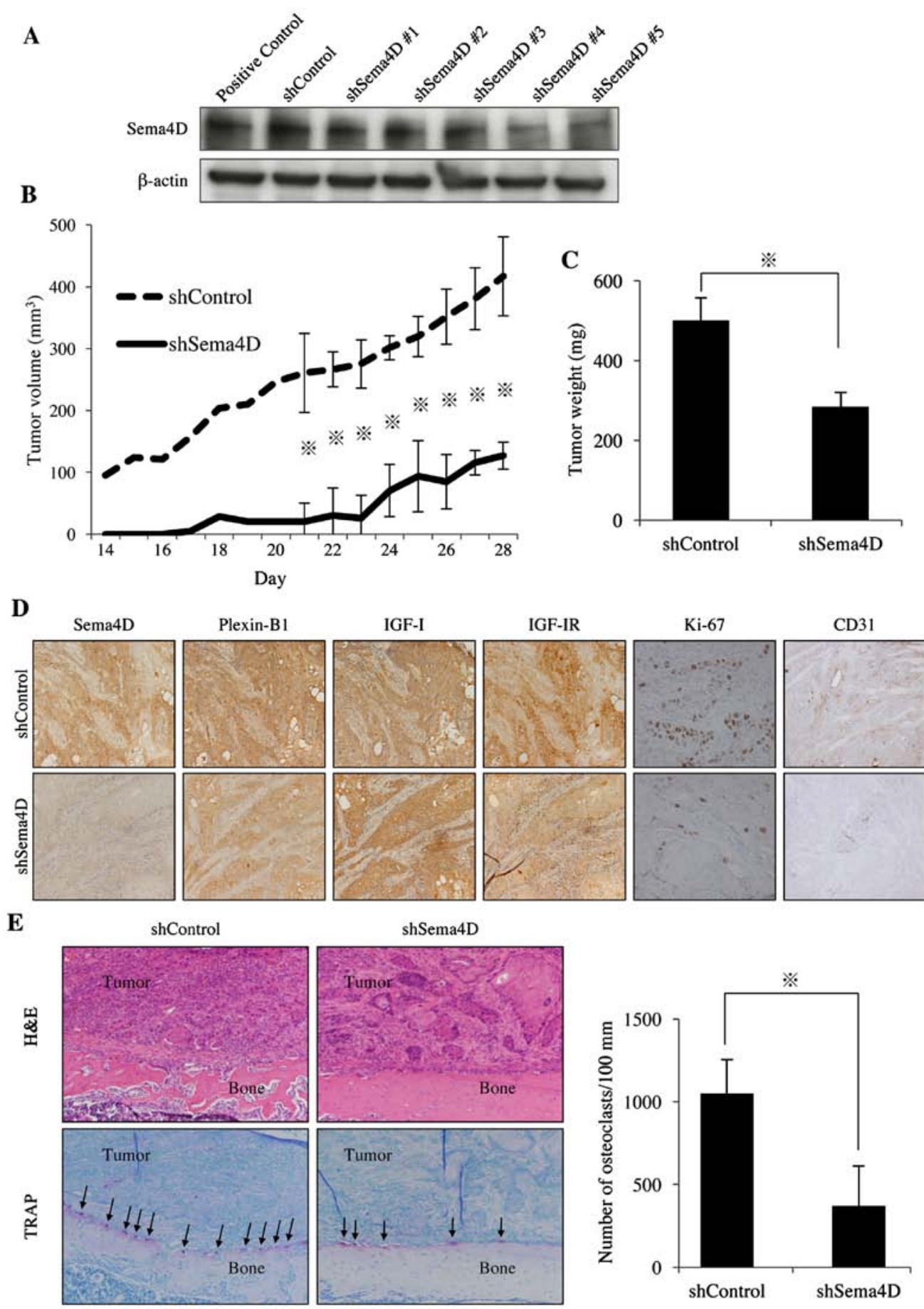

Figure 5. Effect of knockdown of Sema4D on the growth of HSC-2 xenografts and osteoclastogenesis in athymic mice. (A) Knockdown of Sema4D with shRNA was confirmed by immunoblot analysis. (B and C) HSC-2 cells expressing the shControl or shSema4D were inoculated into the paraperiosteal tissue of the tibial metaphysis. Tumor volume and weight were recorded. " $\mathrm{p}<0.05$. (D) IHC staining for Sema4D, Plexin-B1, IGF-I, IGF-IR, Ki-67 and CD31 in tumors from the HSC-2 expressing shControl or shSema4D. Sema4D, Plexin-B1, IGF-I, IGF-IR, CD31: x200 magnification. Ki67: x400 magnification. Ki67-positive and total numbers of cells were counted in 5 randomly selected areas at a magnification of x400. CD31-positive neovessels in each tumor were counted in the five most vascularized areas at a magnification of $\mathrm{x} 200$, and the numbers were averaged. Images shown are from a representative animal of each group. (E) Hematoxylin and eosin (H\&E) and TRAP staining of tumor specimens from the HSC-2 expressing shControl or shSema4D. Magnification, x20. The number of TRAP-positive osteoclasts (arrow) in each group was counted. " $\mathrm{p}<0.05$

osteoclastogenesis without exogenous soluble RANKL and that OPG counteracted Sema4D-induced osteoclastogenesis, supporting the finding that Sema4D increased the RANKL expression in osteoblasts.

Knockdown of Sema4D inhibits bone invasion of xenograft HNSCC in athymic mice. To further investigate whether
Sema4D production by HNSCC cells was responsible for bone invasion, we used shRNA to manipulate Sema4D expression in HNSCC cells. Sema4D shRNAs were purchased and transfected into HSC-2 cells. Immunoblot analysis showed that the Sema4D expression in HSC-2 cells was knocked down by $66.7 \%$ by shSema4D\#4, which were thus used in the subsequent experiments (Fig. 5A). 


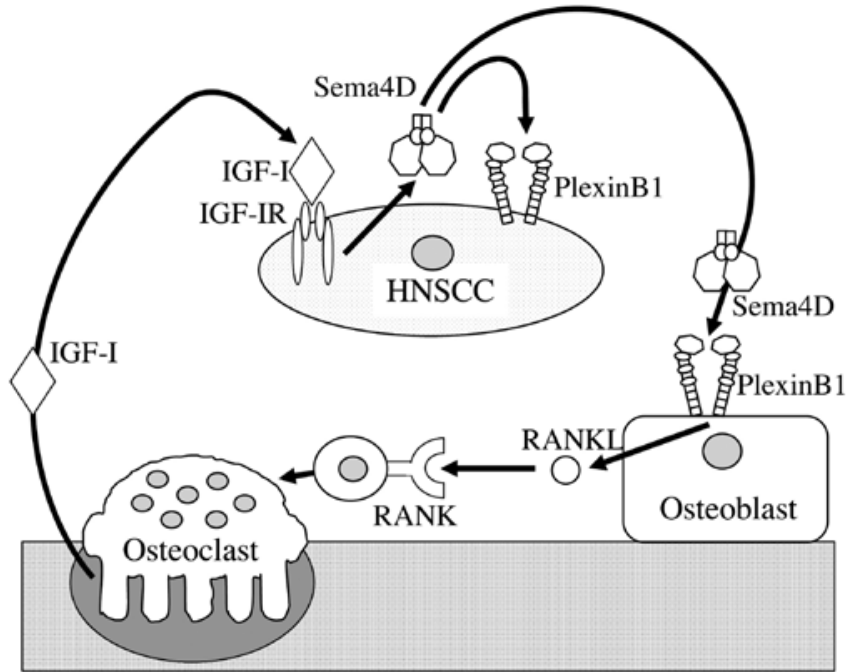

Figure 6. Role of Sema4D in bone invasion in HNSCC. IGF-I, which is released from bone, increases Sema4D secretion in HNSCC cells. Sema4D acts on HNSCC cells in an autocrine manner, which enhances cell activities. Sema4D also regulated RANKL expression in osteoblasts stimulating osteoclastgenesis.

In the animal experiment, the growth rate of xenograft tumors was decreased in Sema4D knockdown group compared to that of the control group (Fig. 5B). The tumor volumes at the end of the experiment (day 28) for the groups treated with shControl and shSema4D were $416.9 \pm 63.8$ and $127.2 \pm 21.9 \mathrm{~mm}^{3}$, respectively, indicating an approximately $69.4 \%$ decrease in tumor growth rate in shSema4D-treated group. The end tumor weights at day 28 for the groups treated with shControl and shSema4D were $500.0 \pm 57.7$ and $283.3 \pm 37.3 \mathrm{mg}$, respectively, indicating an approximately $43.4 \%$ decrease in the tumor growth rate for the shSema4D-treated group (Fig. 5C). These results suggest that knockdown of Sema4D effectively inhibited xenograft tumor growth of HNSCC cells in athymic mice. Our IHC analysis of the tumor specimens showed lower level of Sema4D in the knockdown group, which is consistent with the immunoblot results (Fig. 5A and D). IHC staining with an anti-Ki67 antibody showed that the percentage of Ki67positive cancer cells in the groups treated with shControl and shSema4D were significantly decreased from $55.8 \pm 3.0$ to $15.2 \pm 5.6$, respectively, representing a $72.3 \%$ decrease in cell proliferation (Fig. 5D). Vessel density in the groups treated with shControl and shSema4D, as shown by CD31-positive vessels, was $59.0 \pm 16.7$ and $20.8 \pm 2.5$, respectively, representing a $64.7 \%$ decrease in tumor angiogenesis (Fig. 5D). Knockdown of Sema4D exerted antitumor effects in HNSCC in in vivo experiments via the suppression of cancer cell proliferation and angiogenesis.

To determine whether Sema4D in HNSCC cells influences osteoclastogenesis in vivo, we evaluated the number of TRAPpositive multinucleated osteoclasts in tumor specimens. As shown in Fig. 5E, the numbers of osteoclasts were greatly reduced in the shSema4D-treated group compared to the control group. The quantitative analysis demonstrated a $65.2 \%$ decrease in osteoclast formation following the Sema4D knockdown. Taken together, these results indicate that the expression of Sema4D in HNSCC is important for osteoclastogenesis and for bone invasion (Fig. 6).

\section{Discussion}

Previous research indicated that the axon guidance molecule Sema4D has an important role in cancer progression (19). Sema4D shed by HNSCC cells acts through Plexin-B1 on vascular endothelial cells to promote cell migration and tube formation, which promotes tumor angiogenesis (15). An immunohistochemical analysis of Sema4D in a large tumor sample collection revealed that Sema4D overexpression was present in $80 \%$ of the HNSCCs (15). Sema4D has been shown to act directly on breast (19-21) and ovarian (22) cancer cells, inducing invasion and metastasis.

The results of our present study demonstrate that both Sema4D and Plexin-B1 were expressed in HNSCC cell lines and that Sema4D increased cell proliferation, migration and invasion, suggesting that Sema4D shed by HNSCC cells acts through Plexin-B1 on HNSCC cells themselves in an autocrine manner. A prior study showed that Sema4D activates the small GTPase RhoA, which is related to cytoskeleton reorganization and focal adhesion formation (23). Moreover, Sema4D has also been shown to regulate PYK2 [a nonreceptor tyrosine kinase of the focal adhesion kinase (FAK) family] and modulate cell motility (24). Sema4D causes the AKT and MAPK phosphorylation $(24,25)$, the signaling of which suppresses apoptosis and promotes cell multiplication, respectively. These findings suggest that Sema4D plays a direct role in disease progression of HNSCC, possibly through the signaling pathways mentioned above.

In the bone microenvironment, Sema4D expression in osteoclasts and Plexin-B1 expression in osteoblasts has been observed (14). To date, however, there has been no report describing the expression levels of Sema4D and Plexin-B1 in bone lesions of HNSCC. Our present findings demonstrate that a high expression of Sema4D is present in HNSCCs with bone invasion compared to those without bone invasion. Moreover, Sema4D expression was correlated with IGF-I expression in tumor tissue samples. These findings prompted us to investigate whether IGF-I regulates Sema4D expression.

Of note, IGF-I greatly increased Sema4D expression in HNSCC cells, which is consistent with the results we obtained from the clinical samples. These data support our hypothesis that IGF-I released from bone regulates Sema4D expression and promotes bone invasion of HNSCC. In physiological conditions, IGF-I released from bone is resorbed by osteoclasts, although it was originally stored by osteoblasts, and it induces osteoblast differentiation. In contrast, Sema4D produced by osteoclasts inhibits osteoblast differentiation by inhibiting IGF-I signaling (14). In other words, osteoclasts control bone formation by osteoblasts based on a balance between Sema4D and IGF-I signaling. In osteolytic lesions of HNSCC, a high production of Sema4D by HNSCC cells 'tips the scale' of bone homeostasis in favor of resorption.

Mechanisms underlying the semaphorin enhancement of osteoclastogenesis have been reported (7,26,27). Mice with knock-out of Sema6D receptor plexin-A1 (plexin-A1 ${ }^{-/}$) have been phenotyped as having osteopetrosis due to the inhibition of osteoclastogenesis. In the presence of Sema6D, plexin-A1 forms a complex with TREM2 (triggering receptor expressed on myeloid cells 2) and DAP12 (DNAX-activation protein 12), which promote osteoclastogenesis (26). Sema4D plays a role 
in functions of osteoclasts including spreading, adhesion, migration and resorption through $\beta 3$ integrin subunit downstream signaling (27). Sema4D also promotes IL-8 expression in osteoblasts, which promotes osteoclastogenesis and bone metastasis of breast cancer (7).

In the present study, we observed that the RANKL expression in osteoblasts was upregulated by Sema4D. Strengthening this result, the OPG that was used to counteract RANKL successfully inhibited Sema4D-induced osteoclastogenesis. We also demonstrated that Sema4D shed by HNSCC cells was important for osteoclastogenesis in vivo and for bone invasion, using an animal model. However, we note here that activation of osteoclastogenesis by Sema4D might also be through the above-mentioned factor that has been implicated in a different type of cancer.

Bisphosphonates (28) and an anti-RANKL antibody (denosumab) $(29,30)$ are widely used to manage hypercalcemia of malignancy and skeletal metastases. These drugs target osteoclasts and do not have antitumor activity for cancer cells. The results presented here suggest that targeting Sema4D could be a novel treatment strategy for anti-osteoclastic and antitumor action in HNSCC therapy.

\section{Acknowledgements}

This work was supported by a Grant-in-Aid for Scientific Research (B) (JSPS KAKENHI grant no. JP26293429) to A.S. and a Grant-in-Aid for Scientific Research (C) (JSPS KAKENHI grant no. JP26463004 and JP17K11836) to S.I. from the Ministry of Education, Culture, Sports, Science, and Technology of Japan. The authors would like to thank Ms. Kazuko Funakoshi for the expert technical assistance in histological preparations.

\section{References}

1. Ampil FL, Ghali GE, Caldito G and Hardin JC Jr: Treatment of head and neck cancer with bone or cartilage invasion by surgery and postoperative radiotherapy. J Oral Maxillofac Surg 62: 408-411, 2004

2. Shimo T and Sasaki A: Mechanism of cancer-induced bone destruction: An association of connective tissue growth factor (CTGF/CCN2) in the bone metastasis. Jpn Dent Sci Rev 47: 13-22, 2011.

3. Weilbaecher KN, Guise TA and McCauley LK: Cancer to bone: A fatal attraction. Nat Rev Cancer 11: 411-425, 2011.

4. Shimo T, Kubota S, Yoshioka N, Ibaragi S, Isowa S, Eguchi T, Sasaki A and Takigawa M: Pathogenic role of connective tissue growth factor (CTGF/CCN2) in osteolytic metastasis of breast cancer. J Bone Miner Res 21: 1045-1059, 2006.

5. Luo Y, Raible D and Raper JA: Collapsin: A protein in brain that induces the collapse and paralysis of neuronal growth cones. Cell 75: 217-227, 1993.

6. Kolodkin AL, Matthes DJ and Goodman CS: The semaphorin genes encode a family of transmembrane and secreted growth cone guidance molecules. Cell 75: 1389-1399, 1993.

7. Yang YH, Buhamrah A, Schneider A, Lin YL, Zhou H, Bugshan A and Basile JR: Semaphorin 4D promotes skeletal metastasis in breast cancer. PLoS One 11: e0150151, 2016.

8. Epstein JA, Aghajanian H and Singh MK: Semaphorin signaling in cardiovascular development. Cell Metab 21: 163-173, 2015.

9. Messina A and Giacobini P: Semaphorin signaling in the development and function of the gonadotropin hormone-releasing hormone system. Front Endocrinol (Lausanne) 4: 133, 2013.

10. Kang S, Okuno T, Takegahara N, Takamatsu H, Nojima S, Kimura T, Yoshida Y, Ito D, Ohmae S, You DJ, et al: Intestinal epithelial cell-derived semaphorin 7A negatively regulates development of colitis via $\alpha v \beta 1$ integrin. J Immunol 188: 1108-1116, 2012.
11. Mizui M, Kumanogoh A and Kikutani H: Immune semaphorins: Novel features of neural guidance molecules. J Clin Immunol 29: $1-11,2009$.

12. Shanks K, Nkyimbeng-Takwi EH, Smith E, Lipsky MM, DeTolla LJ, Scott DW, Keegan AD and Chapoval SP: Neuroimmune semaphorin 4D is necessary for optimal lung allergic inflammation. Mol Immunol 56: 480-487, 2013.

13. Moreau-Fauvarque C, Kumanogoh A, Camand E, Jaillard C, Barbin G, Boquet I, Love C, Jones EY, Kikutani H, Lubetzki C, et al: The transmembrane semaphorin Sema4D/CD100, an inhibitor of axonal growth, is expressed on oligodendrocytes and upregulated after CNS lesion. J Neurosci 23: 9229-9239, 2003.

14. Negishi-Koga T, Shinohara M, Komatsu N, Bito H, Kodama T, Friedel RH and Takayanagi H: Suppression of bone formation by osteoclastic expression of semaphorin 4D. Nat Med 17: 1473-1480, 2011.

15. Basile JR, Castilho RM, Williams VP and Gutkind JS: Semaphorin 4D provides a link between axon guidance processes and tumor-induced angiogenesis. Proc Natl Acad Sci USA 103: 9017-9022, 2006.

16. Shimo T, Matsumoto K, Takabatake K, Aoyama E, Takebe Y, Ibaragi S, Okui T, Kurio N, Takada H, Obata K, et al: The role of sonic hedgehog signaling in osteoclastogenesis and jaw bone destruction. PLoS One 11: e0151731, 2016.

17. Goda T, Shimo T, Yoshihama Y, Hassan NM, Ibaragi S, Kurio N, Okui T, Honami T, Kishimoto K and Sasaki A: Bone destruction by invading oral squamous carcinoma cells mediated by the transforming growth factor-beta signalling pathway. Anticancer Res 30: 2615-2623, 2010.

18. Kuroda Y, Hisatsune C, Nakamura T, Matsuo K and Mikoshiba K: Osteoblasts induce $\mathrm{Ca}^{2+}$ oscillation-independent NFATc1 activation during osteoclastogenesis. Proc Natl Acad Sci USA 105: 8643-8648, 2008.

19. Sakurai A, Doçi CL and Gutkind JS: Semaphorin signaling in angiogenesis, lymphangiogenesis and cancer. Cell Res 22: 23-32, 2012.

20. Swiercz JM, Worzfeld T and Offermanns S: ErbB-2 and met reciprocally regulate cellular signaling via plexin-B1. J Biol Chem 283: 1893-1901, 2008 .

21. Worzfeld T, Swiercz JM, Looso M, Straub BK, Sivaraj KK and Offermanns S: ErbB-2 signals through Plexin-B1 to promote breast cancer metastasis. J Clin Invest 122: 1296-1305, 2012.

22. Ye S, Hao X, Zhou T, Wu M, Wei J, Wang Y, Zhou L, Jiang X, Ji L, Chen Y, et al: Plexin-B1 silencing inhibits ovarian cancer cell migration and invasion. BMC Cancer 10: 611, 2010.

23. Capparuccia L and Tamagnone L: Semaphorin signaling in cancer cells and in cells of the tumor microenvironment - two sides of a coin. J Cell Sci 122: 1723-1736, 2009.

24. Basile JR, Afkhami T and Gutkind JS: Semaphorin 4D/plexin-B1 induces endothelial cell migration through the activation of PYK2, Src, and the phosphatidylinositol 3-kinase-Akt pathway. Mol Cell Biol 25: 6889-6898, 2005.

25. Aurandt J, Li W and Guan K-L: Semaphorin 4D activates the MAPK pathway downstream of plexin-B1. Biochem J 394: 459-464, 2006.

26. Kumanogoh A and Kikutani H: Immunological functions of the neuropilins and plexins as receptors for semaphorins. Nat Rev Immunol 13: 802-814, 2013.

27. Dacquin R, Domenget C, Kumanogoh A, Kikutani H, Jurdic P and Machuca-Gayet I: Control of bone resorption by semaphorin 4D is dependent on ovarian function. PLoS One 6: e26627, 2011.

28. Ressler S, Mlineritsch B and Greil R: Zoledronic acid for adjuvant use in patients with breast cancer. Expert Rev Anticancer Ther 11: 333-349, 2011.

29. Stopeck AT, Lipton A, Body J-J, Steger GG, Tonkin K, de Boer RH, Lichinitser M, Fujiwara Y, Yardley DA, Viniegra M, et al: Denosumab compared with zoledronic acid for the treatment of bone metastases in patients with advanced breast cancer: A randomized, double-blind study. J Clin Oncol 28: 5132-5139, 2010.

30. Fizazi K, Carducci M, Smith M, Damião R, Brown J, Karsh L, Milecki P, Shore N, Rader M, Wang H, et al: Denosumab versus zoledronic acid for treatment of bone metastases in men with castration-resistant prostate cancer: A randomised, double-blind study. Lancet 377: 813-822, 2011. 